\title{
ANÁLISE DO "EFEITO TAMANHO” NOS RETORNOS DAS AÇÕES DE EMPRESAS LISTADAS NA BOVESPA
}

\author{
GUSTAVO AMORIM ANTUNES \\ Mestrando em Ciências Contábeis \\ pela FUCAPE - ES \\ E-mail: gaantunes@yahoo.com.br
}

\section{WAGNER MOURA LAMOUNIER}

Prof. Dr. do Depto. de Ciências Contábeis e do

CEPEAD da UFMG - MG

E-mail: Wagner@face.ufmg.br

\author{
AURELIANO ANGEL BRESSAN \\ Prof. Adjunto do CEPEAD da UFMG - MG \\ E-mail: bressan@face.ufmg.br
}

\section{RESUMO}

Neste estudo, o desempenho das ações negociadas na Bovespa foi analisado entre 17 de março de 1998 e 3 de agosto de 2004. Primeiramente, foram feitos testes de estacionariedade para se verificar se as ações seguiram o modelo de passeio aleatório. Verificou-se que todos os retornos foram estacionários. Em relação aos preços, $90 \%$ das ações tiveram preços com raiz unitária, ou seja, apresentaram passeio aleatório no nível. Constatou-se que as $10 \%$ restantes foram ilíquidas. Esses resultados sugerem que o mercado brasileiro é eficiente na forma fraca. Em seguida, a eficiência do mercado brasileiro em sua forma semiforte também foi verificada em relação ao tamanho da empresa, através da estimação de um CAPM condicional. A proxy tradicional para medir o tamanho da empresa é o valor de mercado, mas também foram utilizados o valor patrimonial e o lucro. Os resultados revelam que, independente da proxy utilizada, nenhum portfolio, baseado no tamanho, foi capaz de gerar retornos anormais sistemáticos. Por fim, observou-se que o tamanho está, significativamente, correlacionado com o beta apenas quando se utiliza o valor patrimonial como proxy, porém os coeficientes de correlação apurados foram baixos e apresentaram sinais inconsistentes. Esses resultados, em termos gerais, sugerem, então, que o mercado brasileiro, também, é eficiente na forma semiforte.

Palavras-chave: Efeito Tamanho; CAPM; Mercados Eficientes; Anomalia de Mercado.

\begin{abstract}
This study analyzes the performance of stocks listed on Bovespa - the São Paulo Stock Exchange - between 03/17/1998 and 08/03/2004. First, stationarity was tested in order to check whether these stocks followed the random walk model. The results showed that all returns were stationary. In relation to prices, $90 \%$ of all stock prices revealed a unit root, that is, they followed the random walk model at level. The other $10 \%$ were rarely traded. These findings suggest that the Brazilian stock market is efficient in its weak form. Then, the efficiency of the Brazilian market in its semi-strong form was also tested in terms of the firm's size, by estimating a conditional CAPM. The traditional proxy used to measure a firm's size is the market value. However, other size proxies like book value and profit were also used. The results revealed that, independently of the proxy used, no size portfolio was capable of obtaining systematically abnormal returns. Finally, a significant correlation was found between beta and size only when book value is used as a size proxy. Nevertheless, its coefficients were low and their signals were inconsistent. In general terms, these results suggest that the Brazilian market is also efficient in its semistrong form.
\end{abstract}

Keywords: Size Effect; CAPM; Efficient Capital Markets; Market Anomaly. 


\section{INTRODUÇÃO}

Investigar a eficiência informacional de mercado é importante para se traçar estratégias de investimento. Fama (1991) ressaltou que os trabalhos empíricos sobre o conteúdo informacional dos preços dos ativos financeiros propiciaram o surgimento de estratégias passivas de compra e manutenção de carteiras diversificadas, em resposta à dificuldade de se bater o mercado a partir da análise de informações públicas. Se o mercado acionário é, realmente, eficiente, o investidor não precisa se preocupar em selecionar as ações mais rentáveis, pois todas oferecerão retornos corretamente ajustados aos seus respectivos riscos sistemáticos mensurados pelo beta individual de cada ação. Nesse caso, basta ao investidor diversificar seu portfolio a ponto de fazê-lo acompanhar perfeitamente o índice de mercado (no caso brasileiro, o lbovespa). Feito isso, o investidor só precisa esperar passivamente por um intervalo suficiente de tempo para que ele seja adequadamente remunerado. Esse tempo é necessário para que a relação estatística entre o desempenho do portfolio e o desempenho do índice de mercado se concretize. Caso o mercado não seja eficiente, o investidor deverá identificar aquelas ações mal precificadas para, assim, obter retornos anormais.

Fama (1991), também, afirma que os trabalhos empíricos sobre o conteúdo informacional dos preços dos ativos financeiros incitaram a demanda por análises de desempenho de fundos de investimento para confrontá-los com benchmarks do mercado (CAPM, por exemplo), em resposta às evidências de que informação privada é rara. Se toda informação está realmente inserida eficientemente nos preços, os gestores de fundos de investimentos não seriam capazes de obter retornos anormais sistematicamente. Caso haja evidência de retornos anormais sistemáticos, pode-se suspeitar que o gestor tenha informação privilegiada. Os testes de eficiência fraca, por sua vez, também são relevantes, haja vista o agendamento de negociações permitido pela identificação do efeito-final-de-semana. Mesmo quando esse efeito não é grande o suficiente para permitir transações intensas de compra na segunda-feira e de venda na sexta, as transações já planejadas podem ser adiadas ou antecipadas caso os retornos das segundasfeiras sejam sistematicamente negativos ${ }^{1}$.

\section{OBJETIVOS}

O presente estudo tem como objetivo central verificar se o mercado acionário brasileiro atende aos pressupostos teóricos da hipótese de mercados informacionalmente eficientes. Dito de outra forma, busca-se investigar a eficiência informacional do mercado acionário brasileiro, representado pela Bovespa, nas formas fraca e semiforte. Ao objetivo de avaliação da eficiência fraca, seguem-se dois específicos: verificar a estacionariedade dos retornos das principais ações listadas na Bovespa e identificar se os preços dessas ações seguem o modelo de passeio aleatório.

Quanto à análise de eficiência semiforte, pretende-se investigar a existência do efeito tamanho nos retornos das principais ações da Bovespa, ou seja, verificar se informações sobre o tamanho das firmas são relevantes, ainda não incorporadas pelos preços, sobre os retornos dessas ações. Optou-se por analisar o período de 1998 a 2004, em função do fato de Romaro (2000) ter analisado o período de 1995 a 1998 e encontrado evidências desse efeito. Assim, o presente estudo verifica se aqueles resultados são passíveis de serem estendidos ao período de tempo imediatamente posterior, qual seja, março de 1998 a agosto de 2004.

Aqui, o efeito tamanho será investigado em função de três proxies distintas para mensurar o tamanho das firmas. Esse procedimento visa testar a hipótese de Berk (1997), na qual o efeito tamanho é resultado direto da proxy utilizada para mensurar o tamanho da firma. Ao se empreender o teste de efeito tamanho, será verificada a possibilidade da presença de heterocedasticidade nos resíduos do CAPM tradicional. Será testada, também, a hipótese de Jegadeesh (1992), segundo a qual o efeito tamanho pode estar oculto caso haja correlação entre o beta e o valor de mercado.

Procura-se, portanto, verificar se o mercado de capitais brasileiro atende aos pressupostos da hipótese de eficiência fraca através de dois testes de raiz unitária: um sobre o nível de preços e outro sobre os retornos. Busca-se verificar, ainda, se o mercado de capitais brasileiro atende aos pressupostos da hipótese de eficiência semiforte através do teste de efeito tamanho.

Ao se empreender esse teste, contudo, serão feitos outros três: o primeiro incorpora a possível heterocedasticidade nas séries temporais analisadas no modelo estimado (CAPM Condicional) via modelo GARCH-M; o segundo testa a hipótese de Berk (1997) através de três proxies de tamanho e o terceiro a hipótese de Jegadeesh (1992) através de um de significância estatística da correlação entre o beta estimado e a proxy de tamanho utilizada. Ressalte-se

\footnotetext{
${ }^{1}$ Para mais detalhes acerca dos testes de eficiência, ver Fama (1991).
} 
que o objetivo de todos os testes citados, nesse parágrafo, é unicamente testar a eficiência semiforte.

Assim, o CAPM Condicional é calculado através da metodologia GARCH-M uma única vez. Com o indicador alfa de Jensen apurado, faz-se o teste de efeito tamanho três vezes, um para cada proxy de tamanho. Com o beta apurado, faz-se o teste de correlação três vezes, novamente um para cada proxy de tamanho. Todos esses testes são relevantes, pois tanto a heterocedasticidade quanto as hipóteses de Jegadeesh (1992) e Berk (1997) podem afetar o teste de efeito tamanho e, conseqüentemente, a análise de eficiência informacional.

Em resumo, testa-se a eficiência fraca e a semiforte através de nove testes: dois para se testar a eficiência fraca (ambos de raiz unitária) e sete para se testar a eficiência semiforte (três de efeito tamanho) - com proxies distintas, hipótese de Berk (1997) -, três de correlação com proxies distintas - hipótese de Jegadeesh (1992) - e um de heterocedasticidade - GARCH-M).

\section{REFERENCIAL TEÓRICO}

\subsection{O Efeito Tamanho}

O efeito tamanho, originalmente documentado por Banz (1981), consiste na evidência empírica de que ações de empresas pequenas apresentam maior retorno médio do que as de empresas grandes; evidência essa chamada de anomalia ${ }^{2}$ porque

não há motivo teórico que justifique uma proxy do tamanho da firma ter poder estatístico de explicar diferenças de retorno em uma crosssection, depois de controlada pelo risco. (CHAN et al.,1985, p. 464).

O modelo de precificação de ativos - CAPM prediz que apenas o risco sistemático de uma ação é remunerado pelo mercado, nenhuma outra informação além do beta individual de cada ação é relevante para a formação de seus preços. Assim sendo, a existência do chamado efeito tamanho surgiria como uma anomalia ou uma má especificação do modelo CAPM, uma vez que as evidências empíricas acerca do efeito tamanho indicam "algum risco não medido, ou medido inadequadamente [pelo beta]" (CHAN et al., 1985, p. 464). Esse efeito, também, implicaria ineficiência de mercado, uma vez que os agentes não conseguiriam antecipar esse maior retorno. Depois da ocorrência suficiente do efeito tamanho, os agentes racionais de- veriam ser capazes de revisarem suas expectativas e alterarem seus portfolios em direção às pequenas empresas (maior retorno esperado). Esse aumento da demanda por ações de firmas pequenas (queda na demanda por ações de firmas grandes) elevaria (reduziria) o preço dessas ações hoje, diminuindo (aumentando) o retorno futuro. Assim, o preço se alteraria no exato momento em que os agentes incorporam a informação acerca do efeito tamanho e o retorno futuro das empresas pequenas e grandes seria igual.

Em função desses problemas, alguns trabalhos procuraram elucidar, teoricamente, o efeito tamanho. Fouse (1989) creditou esse efeito ao problema da baixa liquidez das ações de empresas pequenas. Ele argumentou que o investidor pode até estar ciente de determinada informação, mas a falta de liquidez característica da ação impede que ele atue no mercado e insira eficientemente sua informação. Caso isso seja verdadeiro, o efeito tamanho surgiria em função da preferência pela liquidez dos agentes racionais, sendo, portanto totalmente coerente com mercados eficientes.

Em uma outra tese para explicar o efeito tamanho, Berk (1997) argumentou que o efeito tamanho é conseqüência da proxy de tamanho utilizada. Assim, esse efeito não seria uma anomalia, pois;

Não há explicação teórica sobre o porquê de firmas pequenas gerarem retornos maiores porque nenhuma explicação é necessária [já que] a moderna teoria de finanças prediz que o valor de mercado da firma e seu retorno devem ter correlação negativa. (BERK, 1997, p. 12).

Ainda segundo Berk (1997), isso acontece porque não há definição formal acerca do tamanho das empresas. A proxy mais usual, o valor de mercado, consiste em uma estimativa do fluxo de caixa futuro passível de ser gerado pela empresa, trazido a valor presente via taxa de desconto pela fórmula (DAMODARAM, 1997):

$$
\text { Valor }=\sum_{t=1}^{t=n} \frac{C F_{t}}{(1+d)^{t}}
$$

em que:

$n$ é a vida útil do ativo;

$C F_{t}$ é a expectativa de fluxo de caixa futuro da empresa no tempo $t$ e

$d$ é a taxa de desconto (risco) associado à cada empresa.

\footnotetext{
${ }^{2}$ Hazzan (1991) afirma que uma anomalia consiste em uma regularidade não explicada por modelos de risco/retorno ou por diferenças institucionais.
} 
O indicador de valor de mercado, na verdade, comporta dois componentes: ele contém tanto o termo $C F$, que mede a capacidade esperada da empresa de gerar lucros no futuro (definição usual de tamanho) como também possui o termo $(1+d)^{t}$, que mensura o risco referente à materialização desses lucros futuros (mensurado pela taxa de desconto). Desse modo, empresas com idêntica expectativa de lucros futuros (portanto, mesmo tamanho, via definição usual) podem vir a ter valores de mercado diferentes; basta que uma seja mais arriscada que a outra. A mais arriscada terá uma maior taxa de desconto e, portanto, apresentará um menor índice de valor de mercado.

Berk (1997) investigou, então, o efeito tamanho utilizando três proxies distintas: vendas anuais, valor contábil e valor de mercado. Apenas o coeficiente do valor de mercado se revelou significante em seu trabalho. Esse resultado não implica anomalia, apenas revela que o beta não mensura totalmente o risco do ativo que é remunerado pelo mercado. Pode-se explicitar isso matematicamente, por meio das equações:

$$
E\left(R_{i}\right)=\gamma_{0}+\gamma_{1} \bar{\beta}_{i} \quad i=1, \ldots, N .
$$

em que:

$\beta_{i}$ é o risco sistemático verdadeiro remunerado pelo mercado.

$$
\bar{R}_{i}=\tau_{0}+\tau_{1} \beta_{i}+\tau_{2} \operatorname{LnS}_{i}+u_{i,} \quad i=1, \ldots, N
$$

em que:

$\beta_{i}$ é o risco sistemático estimado.

$\mathrm{LnS}_{i}$ é o logaritmo natural da proxy da variável tamanho.

$$
p \lim \tau_{2}=\gamma_{2} \Gamma_{s, \operatorname{LnS} S_{i} \beta_{i}}^{2}
$$

em que:

$p \lim \tau_{2}$ é a probabilidade limite do coeficiente angular $\tau_{2}$ da equação (3) e $\Gamma_{s, L n S_{i} \beta_{i}}^{2}$ mede a proporção da variância residual de $\beta_{i}$ que ê explicada por $L n S_{i}$ depois de descontada a influência de $\beta_{i}$.

\section{Desse modo:}

Se $\left[L n S_{i}\right]$ contém pouca ou nenhuma informação nova sobre $[\beta$ ] que não está já contida em $\left[\beta_{i}\right]$, então, [ $\left.\Gamma_{s, L n S_{i} \beta}^{2}\right]$ e $\tau_{2}$ serão próximos de zero, independentemente de $\gamma$, ser ou não diferente de zero. Porém, se $\left[\Gamma_{s, L n S_{i} \beta_{t}}^{2}\right]$ é próximo de um, o valor de $\tau_{2}$ será próximo ao de $\gamma_{2}$ e os testes serão válidos. (JEGADEESH, 1992, p. 340).
Os resultados de Berk (1997) mostraram que o efeito tamanho surgia apenas quando a proxy utilizada era o valor de mercado. Isso sugere que $L n S_{i}$ contém informação nova e relevante, não contida em $\beta_{i}$, apenas quando a proxy utilizada para medir $L n S_{i}$ é o valor de mercado, em decorrência do valor de mercado medir risco não captado por $\beta_{i}$.

Jegadeesh (1992), por sua vez, analisou as condições nas quais o efeito tamanho pode não ser diretamente observado. O efeito tamanho pode ser ocultado caso haja correlação entre a proxy de tamanho utilizada e o próprio beta $\left(\rho_{\beta S} \cong 1\right)$. Seja a especificação correta do modelo dada por:

$$
E\left(R_{i}\right)=\gamma_{0}+\gamma_{1} \beta_{i}+\gamma_{1} S_{i} \quad i=1, \ldots, N
$$

em que:

$R_{i}$ é o retorno do portfolio $i$ no mês $T$ testado;

$\beta_{i}$ é o seu risco de mercado e

$S_{i}$ é a proxy variável tamanho.

Suponha-se, então, que a correlação entre o beta $(\beta)$ e o tamanho (S) seja unitária, ou seja, $\rho_{\beta S}=1$. Desse modo, o beta de cada ativo, $\beta$, "sozinho explicará virtualmente todas as diferenças encontrada na cross-section dos retornos esperados" (JEGADEESH, 1992, p. 339), mesmo que $\gamma_{2} \neq 0$. Isso é "conseqüência da tendência geral de firmas pequenas terem betas de valores altos e grandes firmas terem betas de valores pequenos" (Op cit., 1992, $p$. 343). No intuito de contornar tal problema, Jegadeesh (1992) montou portfolios com $\rho_{\beta S}$ pequenos em magnitude e verificou que sua amostra do mercado americano revelou a existência do efeito tamanho após essa correção, ou seja, "os resultados indicam que o efeito tamanho não pode ser explicado pelo beta" (Op cit., 1992, p. 349).

\section{METODOLOGIA}

\subsection{Testes de Raiz Unitária}

No presente caso, a aplicação dos testes de estacionariedade visa investigar se os preços passados de uma determinada ação influenciam no seu preço corrente. Isso é feito através da análise da autocorrelação dos resíduos das séries temporais dos preços e dos retornos. Caso haja raiz unitária, pode-se afirmar que o (log do) preço corrente tem relação com os (logs dos) preços passados e que as observações passadas têm influência permanente nos preços futuros. Nesse sentido, ele é um teste do modelo de passeio aleatório.

A estacionariedade fraca, que alicerça, estatisticamente, o conceito de eficiência fraca, exige 
apenas que os dois primeiros momentos (média e autocovariância) sejam constantes ao longo do tempo. Os testes padrões para verificar-se o último tipo de estacionariedade são o Phillips-Perron (PP) e o de Dickey-Fuller Ampliado (ADF). Como a hipótese nula de ambos (PP e ADF) é a existência de raiz unitária, sua rejeição - valor absoluto da estatística $\tau$ (tau) superior ao valor crítico - revela que o processo é estacionário. Os testes descritos, a seguir, permitem a verificação da possibilidade de não-estacionariedade das séries (presença de tendência), assim como identificar se a tendência é do tipo determinística, do tipo estocástica ou uma conjunção das duas. O Teste de Dickey-Fuller Ampliado (ADF), possui a forma geral (LAMOUNIER, 2003):

$$
Y_{t}-Y_{t-1}=\Delta Y_{t}=\mu+\beta t+(\theta-1) Y_{t-1}+\sum_{k=1}^{p-1} \phi_{k} \Delta Y_{t-k}+\varepsilon_{t}
$$

em que:

$\Delta Y_{t}$ é um operador de diferenças;

$\mu$ e $\beta$ verificam a presença de intercepto e tendência linear no modelo;

$(\theta-1)$ verifica a presença de raiz unitária no processo;

$p$ representa o número de defasagens incluídos na equação, para incorporar a possibilidade de que $Y_{t}$ siga um processo auto-regressivo de ordem superior.

O ADF testa, portanto:

a hipótese conjunta $\mu=\beta=(\theta-1)=0$. Se $(\theta-1)=0$, a série possuirá raiz unitária e será considerada como um processo do tipo passeio aleatório. [...]. Se $\mu \neq 0, \beta=0$ e $(\theta-1)=0$, temse que a série apresenta raiz unitária e possui o componente determinístico de intercepto (drift), mas não possui tendência linear determinística, será então um processo do tipo passeio aleatório com intercepto. [...]. Se $\mu \neq 0, \beta \neq 0$ e $(\theta-1)=0$, tem-se que a série apresenta raiz unitária, possui o componente determinístico de intercepto (drift) e possui também a tendência linear determinística, será então um processo do tipo passeio aleatório com tendência linear e intercepto.[...]. Para os casos em que $(\theta-1) \neq 0$ tem-se que a série não possui raiz unitária. (LAMOUNIER, 2003, p.22).
Lamounier (2003) propõe o uso do Teste de Raízes Unitárias de Phillips-Perron ao invés do ADF, pois:

O Teste de Dickey-Fuller Expandido (ADF) nem sempre é o mais indicado para a verificação da presença de raízes unitárias, pois se baseia no pressuposto de que os erros sejam não correlacionados e apresentam variância uniforme, ou seja, pressupõe que $\varepsilon_{\tau} \sim \mathrm{N}\left(0, \sigma^{2}\right)$. (LAMOUNIER, 2003, p. 23).

Ainda segundo Lamounier (2003), o teste PP baseia-se na equação abaixo, que pode ser estimada por MQO:

$$
\Delta Y_{t}=\mu+\rho Y_{t-1}+\varepsilon_{t}
$$

A hipótese nula a ser testada assume que a série $Y$ segue um processo do tipo passeio aleatório. Observa-se que o teste de PP não inclui termos de diferenças defasadas, ao contrário do ADF, mas também pode incorporar termos de tendência determinística e um intercepto. No entanto, devese especificar um termo chamado de defasagem de truncamento ${ }^{3}$, que é relacionado ao número de períodos de correlações seriais que serão incluídas no modelo. Assim, o teste a ser empregado neste trabalho será o de Phillips-Perron.

\subsection{Teste de Efeito Tamanho}

Segundo Damodaran (1997), a eficiência de mercado (em sua forma semiforte) pode ser avaliada mediante estudos de eventos ou de carteiras. Como o objetivo desse estudo é avaliar se uma estratégia de negociação baseada em uma característica observável das empresas (no presente caso, o tamanho) pode levar a ganhos anormais, será empregado o estudo de carteiras. Nesse sentido, será feita uma análise dos retornos médios (média aritmética simples) dos portfolios compostos por empresas grandes e pequenas pertencentes ao índice lbovespa, segmentadas em cinco quintis. $O$ primeiro quintil contém as $20 \%$ maiores empresas e o quinto, as $20 \%$ menores. O mesmo procedimento foi realizado com as empresas selecionadas por valor de mercado e, depois, por valor patrimonial, conforme Berk (1997), e por lucro, ou seja, cinco carteiras de ações, baseadas no tamanho das firmas, foram criadas e rastreadas, ao longo do tempo, para se

\footnotetext{
${ }^{3}$ Para maiores detalhes ver Lamounier (2003).
} 
verificar se, na realidade, há retornos anormais. $\mathrm{O}$ desempenho das carteiras foi avaliado através do modelo de precificação de ativos (CAPM).

Hazzan (1991), afirma que o CAPM é centrado na hipótese de mercado homogêneo e na relação linear risco/retorno, além de pressupor indivíduos avessos ao risco que avaliam o trade-off entre risco e retorno. Assim, agentes racionais maximizam o retorno esperado e minimizam o risco de seus investimentos. Presume-se que todos os investidores possuem o mesmo horizonte de investimento e que eles criam a mesma expectativa a respeito do mesmo retorno esperado, das variâncias e das covariâncias, ou seja, suas expectativas são homogêneas. Além disso, o modelo pressupõe, também, a existência de um ativo livre de risco; que a informação é perfeita; que não existem impostos nem custos de transação e que os ativos são divisíveis. Essa última hipótese permite que o investidor faça decisões marginais. O CAPM prescreve que apenas o risco não-diversificável é remunerado pelo mercado. Esse risco sistemático seria medido pela relação entre as oscilações do ativo e da economia, ou seja, pelo beta através da equação:

$$
E\left(R_{i}\right)=r_{f}+\beta_{i}\left(r_{m}-r_{f}\right) \quad i=1, \ldots, N .
$$

em que:

$r_{f}$ é o retorno do ativo livre de risco;

$r$ é o retorno da carteira de mercado;

$\beta_{i}^{m}$ é o beta do ativo $i$.

As proxies do ativo livre de risco e do retorno de mercado adotadas neste trabalho foram a taxa CDI-over e o índice lbovespa, respectivamente. Vale ressaltar que o Banco Central do Brasil, em sua Resolução $\mathrm{n}^{\circ} 2.829$, de 30.03 .2001 , seção $\mathrm{V}$, art. 46, incisos I e II, estabelece que essas duas variáveis são os parâmetros brasileiros para investimentos em renda fixa e variável, respectivamente.

Para efeito de avaliação de desempenho de portfolios baseados no tamanho das firmas, utilizase o indicador alfa de Jensen:

$$
\left.E\left(R_{i}-r_{f}\right)\right)=\alpha_{i}+\beta_{i}\left(r_{m}-r_{f}\right) \quad i=1, \ldots, N .
$$

em que $\alpha_{i}$ é o alfa do ativo $i$.

O alfa avalia a habilidade de gestores de fundos de selecionar ativos para dentro de seu portfolio. Ele mensura quanto o prêmio pelo risco obtido por um portfolio foi superior ao prêmio predito pelo CAPM. $\mathrm{O}$ alfa pode assumir valores positivos, negativos ou nulos e pode ser ou não estatisticamente significativo. Alfas nulos ou não significativos indicam que o retorno do portfolio foi igual ao predito pela teoria. Alfas positivos (negativos) e significantes indicam que o retorno do portfolio foi superior (inferior) ao predito pelo CAPM.

No presente estudo, o alfa foi o critério utilizado para comparar o desempenho de portfolios compostos por empresas grandes e pequenas, segmentadas em cinco quintis. $O$ primeiro quintil contém as $20 \%$ maiores empresas e o quinto, as $20 \%$ menores. O mesmo procedimento foi realizado com as empresas selecionadas por valor de mercado e por valor patrimonial, conforme Berk (1997), e por lucro. O valor de vendas não foi utilizado, conforme Berk (1997), pois estava disponível apenas para algumas poucas empresas; em seu lugar será utilizado o lucro. Espera-se encontrar valores estatisticamente nulos para o caso de inexistência de efeito tamanho. Caso haja o efeito tamanho, espera-se encontrar coeficientes sistematicamente positivos e significativos para o portfolio que contém as pequenas empresas e coeficientes sistemática e estatisticamente negativos ou nulos para o portfolio que contém as grandes empresas. No caso de inexistência do efeito tamanho, espera-se encontrar alfas estatisticamente nulos para todos os portfolios ou algum alfa esporadicamente diferente de zero.

Para se testar a hipótese de Jegadeesh (1997), será calculada a correlação entre o tamanho, no final de cada ano, de cada uma das ações e os seus respectivos betas. Em seguida, será verificada a significância estatística do valor encontrado através da estatística $t$. A relação linear entre duas variáveis pode ser testada de diversas formas, mas se "o único objetivo de determinado estudo for determinar a existência de correlação, então a Equação [10] é a mais apropriada" (LEVINE et al., 2000, p. 562). Assim, a existência de correlação significativa será testada através da fórmula abaixo:

$$
t=\frac{r-\rho}{\sqrt{\frac{1-r^{2}}{n-2}}}
$$

em que:

A estatística $t$ segue distribuição $t \operatorname{com} n-2$ graus de liberdade;

$n$ é o número de observações;

$r$ é o coeficiente de correlação encontrado;

$\rho$ é o coeficiente de correlação da população.

${ }^{4}<$ www.fipecafi.com.br/cespa/arquivos/legislacao_previ/resolucoes_cmn/resolucao_cmn_2829_01.PDF>, acessado em 27.09.2004. 
O valor $-\rho$ da estatística $t$ foi gerado pelo Excel a partir do comando distt. Como a hipótese nula desse teste assume que não existe correlação, $p \leq 0,10$ indica que $\rho \neq 0$ ao nível de $10 \%$ de significância.

\subsection{O Modelo GARCH-M}

É praticamente fato estilizado em finanças que a volatilidade de ativos financeiros apresenta autocorrelação (GUJARATI, 2000). Para contornar esse problema, estimou-se o modelo GARCH-M da seguinte forma: primeiramente, estimou-se o CAPM tradicional, conforme equação (9). Posteriormente, estimou-se a volatilidade condicional por meio de um processo GARCH $(1,1)$, conforme equação abaixo:

$$
h_{t}=w_{0}+\sum_{i=1}^{p} \phi_{i} \varepsilon_{t-1}^{2}+\sum_{i=1}^{q} \theta_{i} h_{t-j}+v_{t}
$$

em que:

$h_{t}$ é a volatilidade condicional no tempo $t$;

$w_{0}$ é o termo médio da volatilidade condicional;

$\phi_{i}$ é o componente auto-regressivo dos resíduos quadráticos (parâmetro ARCH);

$\varepsilon_{t-1}^{2}$ é o quadrado dos resíduos do CAPM tradicional com uma defasagem;

$\theta_{i}$ é o parâmetro do componente auto-regressivo da volatilidade (parâmetro GARCH);

$h_{t-q}$ é a volatilidade defasada na ordem $q$;

$v_{t}^{t-q}$ e um processo ruído branco $[\mathrm{N} \sim(0,1)]$.

Em seguida, como último passo, a volatilidade condicional $h_{t}$ será retornada à equação original - equação (9), formando um CAPM condicional, à medida que incorpora o componente de variância condicional como variável explicativa dos retornos em excesso dos ativos.

Araújo et al. (2003) afirma que a forma mais simples do modelo (11) (GARCH de ordem $p=1$ e $q=1$ ) é funcional para descrever o comportamento da volatilidade condicional de uma série temporal. Nesse sentido, por ser mais parcimonioso que modelos GARCH de ordens superiores essa formulação será utilizada para a estimação do CAPM condicional caso se verifique presença de heterocedasticidade nos resíduos do CAPM tradicional.

\subsection{Dados Utilizados}

Os dados foram coletados junto à base de dados LAFIS, processados a partir do software EXCEL e analisados por meio do software E-VIEWS 4.1. Todas as empresas foram ordenadas, ano a ano, de 1998 a 2004, segundo o tamanho de cada uma no final do ano anterior. Foram usadas três proxies para medir o tamanho das firmas: o tradicional valor de mercado, bem como o valor contábil e o lucro.

Os preços utilizados foram os preços diários de fechamento das ações das empresas e os indicadores (quantidade de ações, lucro por ação e valor contábil por ação), todos valores referentes ao final do ano. Ressalta-se que ambas as informações (preços e indicadores) foram previamente deflacionadas pelo IPCA e corrigidas para proventos pelo próprio site (<www.lafis.com.br $>$ ) na qual essas informações foram coletadas. Todos os preços foram obtidos em 05.08.2004 e todos os indicadores, em 06.08.2004. O LAFIS disponibiliza, apenas, os dados referentes aos dias em que determinada ação é efetivamente negociada, sendo que não há valores (preço de fechamento) para os dias em que a ação não é negociada. Nesses casos, considerou-se que o retorno do ativo foi nulo na ausência de negociação, ou seja, os missing values foram preenchidos utilizando-se a última cotação anterior. Dito em outras palavras, nos dias em que não houve negociação de determinada ação, tomou-se como preço de fechamento o preço de fechamento do dia imediatamente anterior. Esse procedimento foi preferido à interpolação, pois a mesma assume que o mercado avalia o retorno médio (inconsistente com o uso de retornos diários). Com relação ao valor de mercado de cada empresa, este foi calculado pela fórmula:

$$
p_{i, t}^{o n} \cdot q_{i, t}^{o n}+p_{i, t}^{p n} \cdot q_{i, t}^{p n}
$$

em que:

$p_{i, t}^{o n}$ é a última cotação do ano da ação do tipo ON da empresa $i$ no ano $t$;

$p_{i, t}^{p n}$ é a última cotação do ano da ação do tipo PN, PNA ou PNB da empresa $i$ no ano $t$, dependendo se a ação em questão é do tipo 4, 5 ou 6;

$q_{i, t}^{o n}$ é a quantidade de ações do tipo ON da empresa $i$ no ano $t$

$q_{i, t}^{p n}$ é a quantidade de ações do tipo PN da empresa $i$ no ano $t$.

Os valores patrimoniais e os lucros de cada empresa foram calculados multiplicando-se, respectivamente, os valores patrimoniais por ação e os lucros por ação pela quantidade total de ações da empresa no final de cada ano.

Inicialmente, foram selecionadas todas as 76 ações que participaram da composição da carteira teórica de setembro a dezembro do índice lbovespa de cada ano entre 1997 e 2003, no intuito de se evitarem os problemas da pouca freqüência de 
transações. Isso porque o não-sincronismo na negociação diária das ações,

induz uma auto-correlação positiva num índice de ações formado por elas [...], principalmente quando a ação é pouco negociada, ao se estimar seu beta, a covariância dos retornos dessa ação com os retornos do índice de mercado será subestimada, fazendo com que o beta também seja subestimado (COSTA JÚNIOR et al., 2000, p. 86-87).

Em seguida, foram excluídas, ano a ano, aquelas empresas cujas ações não tinham indicadores disponíveis sobre o ano anterior ao ano em análise. Assim, o número de ações analisadas em cada ano variou conforme a disponibilidade dos dados (indicadores de tamanho), como se vê na TAB. 1.

\section{TABELA 1 - 0 tamanho das amostras anuais - 1998-2004}

\begin{tabular}{l|c|c|c|c|c|c|c}
\hline Ano & 1998 & 1999 & 2000 & 2001 & 2002 & 2003 & 2004 \\
\hline $\mathbf{N}^{\circ}$ de ações & 25 & 38 & 43 & 58 & 62 & 67 & 64 \\
\hline Observações & 194 & 246 & 248 & 246 & 249 & 249 & 147 \\
\hline
\end{tabular}

Fonte: Resultados desta pesquisa

Vale notar que os portfolios são montados anualmente contendo todas as ações pertencentes ao índice lbovespa do final de cada ano, ou seja, o portfolio do ano $t$ pode conter empresas que não compuseram o índice lbovespa nos anos anteriores ou posteriores. Assim, evitou-se analisar apenas as ações sem interrupções durante todo o período analisado, mar./1998 a jul./2004, para não incorrer no viés de sobrevivência descrito abaixo:

A eliminação, sem nenhum critério, destas ações [cotadas descontinuamente] pode acarretar algum viés durante a análise dos retornos ou outra variável em foco, pois estas ações poderiam apresentar certas características que poderiam alterar significativamente os resultados. (COSTA JÚNIOR; O'HANLON, 1991, p. 62).

\section{RESULTADOS E DISCUSSÃO}

Os resultados descritos, a seguir, referem-se à amostra coletada junto à base de dados LAFIS que contém todas as ações que participaram da composição da carteira teórica de setembro a dezembro do índice lbovespa de cada ano entre 1997 e 2003 e cujas ações apresentavam indicadores disponíveis sobre o ano anterior ao ano em análise.

Ainda, uma vez que se detectou heterocedasticidade nas séries temporais (resultados descritos mais adiante), a estimação do CAPM tradicional foi considerada inapropriada. Assim, todos os resultados apresentados já são os referentes à estimação do CAPM condicional via GARCH-M. A seguir, apresenta-se o resultado de cada um dos nove testes realizados. Adianta-se que, apesar da multiplicidade de testes, todos foram empreendidos sobre a mesma base de dados.

\subsection{Resultados do Teste de Estacionariedade}

Primeiramente, o teste de Phillips-Perron foi feito para as séries de retornos de todas as ações selecionadas. Rejeitou-se a existência de raiz unitária para os retornos, mesmo ao nível de $1 \%$ de significância, em todos os casos. Isso significa que os retornos apresentaram estacionariedade, o que condiz com a forma fraca da Hipótese de Mercados Eficientes (HME). Em seguida, o mesmo teste foi empregado para o nível de preços. A TAB. 2 mostra os valores- $p$ encontrados nos testes de Phillips-Perron feitos para os níveis de preços de todas as ações selecionadas. Esses valores expressam a que nível de significância a hipótese nula (existência de raiz unitária) não pode ser rejeitada, ou seja, se o valor- $p$ for maior que 0.1 , tem-se que não se pode rejeitar a hipótese nula ao nível de $10 \%$ de significância. O modelo de passeio aleatório prediz que os preços devem possuir raiz unitária, de modo que sigam um passeio aleatório. Observe-se que só 7 valores não foram significativos (em itálico) ao nível de 10\% de significância.

No intuito de se confirmar a estacionariedade dos preços desses 7 ativos, o teste ADF foi empregado para essas mesmas 7 séries de preços. Em seguida, as séries de preços foram refeitas, utilizando-se a técnica de interpolação linear, ao invés de utilizar o preço anterior para os dias sem negociação, no intuito de se verificar se essa técnica poderia alterar os resultados. 
TABELA 2 -Teste PP para o nível de preços

\begin{tabular}{l|c|l|l|l|l|l|l}
\hline \multicolumn{1}{c|}{ Ativo } & valor-p & Ativo & valor-p & Ativo & valor-p & Ativo & valor-p \\
\hline Ibov & 0.6393 & ITSA4 & 0.8756 & TBLE3 & 0.7171 & BRKM5 & 0.8174 \\
\hline BBDC4 & 0.496 & EBTP4 & 0.7189 & BELG4 & 0.9997 & CLSC6 & $0.0417^{* *}$ \\
\hline BRAP4 & 0.1071 & TSPP4 & 0.6125 & VCPA4 & 0.9793 & CPSL3 & 0.9999 \\
\hline BBAS3 & 0.9277 & BRTP4 & 0.1041 & TMCP4 & 0.1605 & TLCP4 & 0.3923 \\
\hline BBAS4 & $0.0750^{* * *}$ & SBSP3 & 0.2962 & PALF3 & 0.2663 & CGAS5 & 0.8563 \\
\hline PETR4 & 0.748 & INEP4 & $0.0750^{* * *}$ & TCSL4 & 0.2475 & DURA4 & 0.5928 \\
\hline CSNA3 & 0.9996 & CRUZ3 & 0.9813 & ELPL4 & 0.1132 & EBEN4 & $0.0001^{* * *}$ \\
\hline GGBR4 & 1 & ARCZ6 & 0.9415 & CSTB4 & 0.9998 & GETI4 & 0.7735 \\
\hline PTIP4 & 0.5133 & TMAR5 & 0.2339 & CRTP5 & 0.3322 & PMAM4 & 0.1619 \\
\hline VALE5 & 0.9242 & BRTO4 & 0.4088 & TNEP4 & 0.1423 & BMTO4 & 0.2017 \\
\hline ELET3 & 0.1351 & BESP4 & 0.9028 & BRDT4 & 0.6957 & TDBH4 & 0.3272 \\
\hline PLIM4 & 0.6769 & ELET6 & 0.1833 & SCON4 & 0.9996 & UNIP6 & 0.9999 \\
\hline ITAU4 & 0.6553 & GEPA4 & 0.0181 & USIM6 & 0.4118 & TPRC6 & 0.2093 \\
\hline AMBV4 & $0.0488^{* *}$ & TCOC4 & 0.3156 & USIM4 & 0.9996 & REPA4 & $0.0111^{* *}$ \\
\hline TLPP4 & 0.4789 & USIM5 & 0.9996 & KLBN4 & 0.5394 & EMAE4 & 0.4809 \\
\hline TNLP4 & 0.1457 & CMIG4 & 0.4722 & LIGH3 & 0.1971 & LIPR3 & $0.0022^{* * *}$ \\
\hline EMBR3 & 0.674 & CPLE6 & 0.5242 & ACES4 & 0.953 & & \\
\hline EMBR4 & 0.6814 & CESP4 & 0.1489 & TRPL4 & 0.4988 & & \\
\hline
\end{tabular}

Fonte: Resultados desta pesquisa.

Nota: Ações ordenadas em função do Valor de Mercado médio do período analisado.

*significância de 10\% $\quad$ ** significância de 05\% $\quad$ *** significância de 01\%

A TAB. 3 revela a posição de cada ação no ranking de valor de mercado médio do período e o $p$ value dos testes de raiz unitária. Caso esse valor seja menor que 0.1 , pode-se rejeitar a hipótese de que os preços tenham raiz unitária. Observa-se que, independente do teste de raiz unitária e da técnica utilizada para preencher os dias sem negociação, os preços dessas 7 ações são estacionários. Ainda, que esses resultados não são função do valor de mercado das empresas (medido, aqui, pelo VM médio de todo período), uma vez que essas ações pertencem a empresas de variadas posições no ranking de VM médio.

TABELA 3 - Testes de estacionariedade complementares

\begin{tabular}{|c|c|c|c|c|c|c|c|c|}
\hline \multirow[b]{2}{*}{ Ativo } & \multicolumn{8}{|c|}{ Testes de estacionariedade complementares } \\
\hline & BBAS4 & AMBV4 & INEP4 & GEPA4 & CLSC6 & EBEN4 & REPA4 & LIPR3 \\
\hline Posição VM médio & $4^{\circ}$ & $13^{\circ}$ & $23^{\circ}$ & $30^{\circ}$ & $55^{\circ}$ & $60^{\circ}$ & $67^{\circ}$ & $69^{\circ}$ \\
\hline ADF preço anterior & $0,0683^{*}$ & $0,0459^{\star *}$ & $0,0032^{\star \star *}$ & $0,0035^{\star \star \star}$ & $0,0365^{\star \star}$ & $0,0006^{\star \star \star}$ & $0,0102^{\star \star}$ & $0,0137^{\star \star}$ \\
\hline PP interpolação & $0,0750^{\star}$ & $0,0488^{\star \star}$ & $0,0075^{\star \star \star}$ & $0,0183^{\star \star}$ & $0,0417^{\star *}$ & $0,0001^{\star \star \star}$ & $0,0113^{\star \star}$ & $0,0022^{\star \star \star}$ \\
\hline
\end{tabular}

Fonte: Resultados desta pesquisa.

Nota: * significância de $10 \% \quad$ ** significância de $05 \% \quad$ *** significância de $01 \%$ 
Esses testes sugerem uma "janela de ineficiência", ao passo que retornos superiores podem ser obtidos através de técnicas elaboradas para se produzir um melhor estimador. Contudo, isso se deve à baixa liquidez dessas 7 ações. A TAB. 4 mostra a participação percentual de cada uma dessas ações na carteira teórica do lbovespa no final de cada ano entre 1998 e 2003 (as informações pertinentes ao ano de 2004 referem-se ao final do primeiro semestre). $O$ código $n c$ (não cotada) indica que a ação não era negociada no ano correspondente e o código aus (ausente) indica que a ação não participou do índice lbovespa em dezembro do ano correspon- dente. Observa-se que todas possuem liquidez muito baixa e estiveram ausentes do índice lbovespa em pelo menos um ano. A REPA4, por exemplo, não participou do lbovespa nenhuma vez entre 1998 e $2004^{5}$. A BBAS4 foi a mais líquida dentre as 07, mas verificou-se que ela foi, em média, apenas a $37^{\mathrm{a}}$ ação mais negociada na Bovespa entre 1998 e $2002^{6}$. Assim, pode-se concluir que algumas ações não se comportaram conforme o modelo de passeio aleatório. Todavia, em função da baixa liquidez dessas ações a "janela de ineficiência", provavelmente, não pôde ser aproveitada pelos investidores para obter ganhos anormais com essas ações.

TABELA 4 - Testes complementares

\begin{tabular}{l|r|r|r|r|r|r|r|r}
\hline & \multicolumn{9}{|c}{ Participação na Carteira Teórica do Ibovespa do final do período - (em \%) } \\
\hline Ano & BBAS4 & AMBV4 & INEP4 & GEPA4 & CLSC6 & EBEN4 & REPA4 & LIPR3 \\
\hline 1998 & 2,2660 & nc & 1,3800 & nc & 0,6360 & 0,1470 & aus & 0,4830 \\
\hline 1999 & 1,5980 & aus & 0,9090 & 0,7210 & 0,9730 & aus & aus & aus \\
\hline 2000 & 0,9680 & aus & 0,7050 & aus & 1,0840 & aus & aus & aus \\
\hline 2001 & 1,3000 & 1,3810 & 0,3720 & aus & 0,4720 & aus & aus & aus \\
\hline 2002 & 1,4701 & 1,7461 & 0,2013 & aus & 0,5351 & aus & aus & aus \\
\hline 2003 & nc & aus & aus & aus & aus & aus & aus & aus \\
\hline $2004^{*}$ & nc & 2,7900 & aus & aus & 0,6430 & aus & aus & aus \\
\hline Média & 1,5204 & 0,9862 & 0,5096 & 0,1202 & 0,6204 & 0,0210 & 0,0000 & 0,0690 \\
\hline
\end{tabular}

Fonte: Bovespa

Nota: A média aqui indicada refere-se à média dos anos em que a ação era negociada na Bovespa, ou seja, os períodos assinalados por nc não foram contabilizados.

* informações referentes ao final do primeiro semestre.

\subsection{Resultados dos Componentes do Modelo GARCH}

A presença de autocorrelação dos resíduos significa que os modelos da família GARCH são mais apropriados (GUJARATI, 2000, p. 440). Por esse ser fato estilizado em finanças, o CAPM condicional foi estimado a partir da estrutura do modelo GARCH, no intuito de incorporar os efeitos da volatilidade condicional e permitir uma estimação mais precisa dos parâmetros do CAPM, mais precisamente, do alfa. Os resultados obtidos mostram a significância dos componentes ARCH e GARCH, ou seja, corroboram o fato estilizado já citado.

Após a estimação do CAPM condicional, verificou-se que 52 dos 105 parâmetros $\mathrm{ARCH}$ estimados, anualmente, foram significativos ao nível de $10 \%$ de significância. No longo prazo, todavia, observou-se que todos os parâmetros $\mathrm{ARCH}$ foram significativos ao nível de 1\% (TAB. 5). Esse resultado indica que a volatilidade dos retornos apresenta uma estrutura condicional.

\footnotetext{
${ }^{5}$ Essa ação só participou da amostra analisada porque ela compunha o Ibovespa em dezembro de 1997
}

${ }^{6}$ A BBAS4 não foi mais negociada a partir de 2003, como mostra a tabela 4. 
O componente GARCH, por sua vez, mostrouse ainda mais significativo em todos os três grupos de portfolios montados. Dos 105 parâmetros estimados, 76 foram significativos ao nível de $10 \%$. No longo prazo, todos os parâmetros GARCH revelaram-se significativos ao nível de 1\% (TAB. 6). Esse resultado indica que a volatilidade dos retornos pode ser modelada incluindo-se termos GARCH.

\subsection{Resultados dos Testes de Efeito Tamanho ${ }^{7}$}

\subsubsection{Grupo de Valor de Mercado (VM)}

Após a estimação do CAPM condicional, não se verificou o efeito tamanho ao se utilizar, como proxy de tamanho, a variável valor de mercado. A TAB. 7 mostra que, no grupo de valor de mercado, todos quintis apresentaram algum alfa significativo a $10 \%$ no curto prazo. Contudo, essa significância não é recorrente, uma vez que ela ocorre apenas em um único ano isolado em cada caso. Ainda, contrariando a predição do efeito tamanho, o quinto quintil (que comporta as menores empresas) apresentou a menor quantidade de alfas positivos (apenas um) e a única significância encontrada foi para um alfa negativo, logo, o quinto quintil revelou o pior desempenho no curto prazo. No longo prazo, apenas o quarto e quinto quintil apresentaram alfas significativos. Contudo, o quinto quintil apresentou alfa negativo, o que indica seu pior desempenho também no longo prazo.

\section{TABELA 5 - Parâmetros ARCH estimados para 1998-2004}

\begin{tabular}{|c|c|c|c|c|c|}
\hline \multirow[b]{2}{*}{ Proxy ${ }^{\star \star \star}$} & \multicolumn{5}{|c|}{ Quintis } \\
\hline & 1 & 2 & 3 & 4 & 5 \\
\hline Lucro & 0.0049 & 0.0584 & 0.4736 & 0.0644 & 0.0465 \\
\hline V. Mercado & 0.0317 & 0.0872 & 0.1004 & 0.0410 & 0.3880 \\
\hline V. Patrim. & 0.1008 & 0.0472 & 0.1827 & 0.0114 & 0.0543 \\
\hline
\end{tabular}

Fonte: Resultados desta pesquisa.

Nota: ${ }^{* \star *}$ todos os parâmetros foram significativos a $1 \%$

\section{TABELA 6 - Parâmetros GARCH estimados para 1998-2004}

\begin{tabular}{l|c|c|c|c|c}
\hline & \multicolumn{5}{|c}{ Quintis } \\
\hline Proxy $^{\star \star *}$ & 1 & 2 & 3 & 4 & 5 \\
\hline Lucro & 0.9926 & 0.9334 & 0.6392 & 0.9277 & 0.9521 \\
\hline V. Mercado & 0.9650 & 0.9042 & 0.8822 & 0.9592 & 0.6692 \\
\hline V. Patrim. & 0.8986 & 0.9483 & 0.9128 & 0.9875 & 0.9405 \\
\hline
\end{tabular}

Fonte: Resultados desta pesquisa

*** todos os parâmetros foram significativos a $1 \%$

TABELA 7 - Alfas dos portfolios VM

\begin{tabular}{|c|c|c|c|c|c|}
\hline \multirow[b]{2}{*}{ Ano } & \multicolumn{5}{|c|}{ Quintis baseados no Valor de Mercado } \\
\hline & 1 & 2 & 3 & 4 & 5 \\
\hline 1998 & -0.0005 & $-0.0021^{*}$ & -0.0003 & -0.0012 & 0.0018 \\
\hline 1999 & 0.0006 & 0.0009 & $-0.0016^{\star \star \star}$ & -0.0001 & -0.0012 \\
\hline 2000 & -0.0001 & -0.0004 & -0.0004 & -0.0002 & -0.0014 \\
\hline 2001 & 0.0005 & -0.0001 & 0.0005 & 0.0012 & -0.0004 \\
\hline 2002 & 0.0004 & -0.0001 & -0.0001 & 0.0007 & $-0.0018^{\star \star \star}$ \\
\hline 2003 & $0.0006^{\star}$ & -0.0004 & 0.0003 & $0.0010^{\star}$ & -0.0004 \\
\hline 2004 & -0.0003 & -0.0002 & 0.0001 & 0.0005 & -0.0004 \\
\hline 1998-2004 & 0.0001 & -0.0001 & 0.0002 & $0.0008^{\star \star \star}$ & $-0.0012^{\star \star \star}$ \\
\hline \multicolumn{6}{|c|}{ Fonte: Resultados desta pesquisa. } \\
\hline \multicolumn{2}{|c|}{ Nota: *significância de 10\% } & \multicolumn{2}{|c|}{ **significância de 05\% } & \multicolumn{2}{|c|}{ *** significância de 01\% } \\
\hline
\end{tabular}

\footnotetext{
${ }^{7}$ A estimação do CAPM tradicional produziu resultados semelhantes aos da versão condicional. Assim, serão apresentados apenas os resultados dessa última em função da existência de heterocedasticidade condicional na versão tradicional.
} 
A não existência do efeito tamanho não se deveu à correlação entre o tamanho e o beta ${ }^{8}$. A TAB. 8 mostra que houve correlação estatisticamente significativa (ao nível de 10\%) entre o LnVM e o beta apenas em um ano. Mesmo assim, a correlação en- contrada nesse ano é de apenas 0.25 , ou seja, muito distante da unidade. Com isso, pode-se afirmar que o argumento de Jegadeesh (1992) não é válido para a amostra, aqui, analisada.

TABELA 8 - Correlação, ano a ano, entre LnVM e o beta

\begin{tabular}{l|c|c|c|c|c|c|c}
\hline & \multicolumn{7}{c}{ Ano } \\
\hline Proxy & 1998 & 1999 & 2000 & 2001 & 2002 & 2003 & 2004 \\
\hline LnVM & 0.2533 & 0.1385 & -0.1726 & 0.1652 & $0.2510^{\star \star}$ & -0.0617 & 0.0748 \\
\hline g. I. & 23 & 36 & 41 & 56 & 60 & 65 & 63 \\
\\
Fonte: Resultados desta pesquisa. \\
Nota: ${ }^{* *}$ significância de 05\%
\end{tabular}

\subsubsection{Grupo de Valor Patrimonial (VP)}

Após a estimação do CAPM condicional, observou-se que os portfolios baseados no valor patrimonial não revelaram alfas sistematicamente diferentes de zero, ou seja, não se observou o efeito tamanho quando o tamanho da firma foi calculado a partir do seu respectivo valor patrimonial. No curto prazo, três obtiveram alfa significativo a $10 \%$. Contudo, a sua significância é eventual, ocorrendo apenas em um ano isolado no caso dos quintis 03 e
04. O quinto quintil apresentou alfa significativo em dois anos, porém ambos se mostraram negativos. Além disso, o quinto quintil é o que possui o menor número de alfas positivos (apenas dois), e é o único portfolio a apresentar alfa negativo e significativo, ou seja, ele foi o que apresentou o pior desempenho no curto prazo. No longo prazo apenas os quintis 03 e 05 apresentaram alfas significativos. Porém, o alfa do quinto quintil apresentou-se negativo; notese que esse foi o único alfa negativo no longo prazo. A TAB. 9 apresenta esses resultados.

TABELA 9 - Alfas dos portfolios VP

\begin{tabular}{l|c|c|c|c|c}
\hline & \multicolumn{5}{|c}{ Quintis baseados no Valor Patrimonial } \\
\hline Ano & 1 & 2 & 3 & 4 & 5 \\
\hline 1998 & -0.0006 & -0.0008 & -0.0018 & -0.0013 & -0.0023 \\
\hline 1999 & -0.0010 & 0.0002 & 0.0007 & -0.0002 & 0.0013 \\
\hline 2000 & 0.0002 & -0.0002 & -0.0002 & 0.0004 & -0.0002 \\
\hline 2001 & 0.0008 & -0.0004 & 0.0008 & 0.0002 & $-0.0019^{\star \star *}$ \\
\hline 2002 & 0.0004 & 0.0003 & 0.0004 & 0.0000 & $-0.0025^{\star \star *}$ \\
\hline 2003 & -0.0003 & 0.0001 & $0.0015^{\star \star}$ & $0.0023^{\star \star *}$ & 0.0008 \\
\hline 2004 & -0.0001 & 0.0011 & 0.0005 & 0.0004 & -0.0001 \\
\hline $1998-2004$ & 0.0002 & 0.0001 & $0.0016^{\star \star \star}$ & 0.0003 & $-0.0007^{\star}$ \\
\hline
\end{tabular}

Fonte: Resultados desta pesquisa

Nota: *significância de 10\% $\quad$ ** significância de 05\% $\quad$ *** significância de $01 \%$

${ }^{8}$ Calculado por meio da divisão da covariância do ativo com o mercado pela variância do mercado. 
A TAB. 10 mostra que houve correlação entre LnVP significativa mesmo ao nível de $5 \%$ em quase todos os anos (a exceção foi o ano 2000). Todavia, o sinal dessa correlação não foi homogêneo, pois, em 2004, se apresentou negativa. Além disso, apenas dois coeficientes foram maiores que 0,50 . Jegadeesh
(1992) argumentou que o valor dessa correlação deveria ser alto, próximo da unidade, para que o efeito tamanho fosse ocultado. Desse modo, esses valores parecem não ter influenciado os resultados descritos anteriormente sobre a não existência do efeito tamanho nos portfolios baseados no valor patrimonial.

TABELA 10 - Correlação, ano a ano, entre LnVP e beta

\begin{tabular}{l|c|c|c|c|c|c|c}
\hline & \multicolumn{7}{c}{ Ano } \\
\hline Proxy & 1998 & 1999 & 2000 & 2001 & 2002 & 2003 & 2004 \\
\hline LnVM & $0.6955^{\star \star \star}$ & $0.5513^{\star \star \star}$ & -0.1105 & $0.3261^{\star \star}$ & $0.41831^{\star \star \star}$ & $0.3082^{\star \star}$ & $-0.3930^{\star \star \star}$ \\
\hline g. I. & 23 & 36 & 41 & 56 & 60 & 65 & 63 \\
Fonte: Resultados desta pesquisa. \\
Nota: ${ }^{* \star}$ significância de 1\%
\end{tabular}

\subsubsection{Grupo de Lucro (L)}

Após a estimação do CAPM condicional, apurou-se que os portfolios baseados no lucro não revelaram efeito tamanho no curto prazo. A TAB. 11 mostra que quatro quintis obtiveram alfa significativo a $10 \%$. Todavia, novamente a significância ocorreu em anos isolados. O quinto quintil, novamente, possui o menor número de alfas positivos (apenas três) e apresentou alfa negativo e significativo a $10 \%$ de significância; o que indica seu pior desempenho no curto prazo. Esse sugere que não há indícios de efeito tamanho no curto prazo. No longo prazo, po- rém, parece ter ocorrido o efeito tamanho de modo invertido. Os portfolios que continham as maiores firmas obtiveram alfas positivos e significativos a $10 \%$; os alfas dos dois primeiros quintis foram significativos inclusive ao nível mais restrito de $5 \%$.

Ressalte-se que a magnitude desse efeito não foi alterada pela correlação ${ }^{9}$ entre lucro médio e o beta. A TAB. 12 mostra que essa correlação não foi estatisticamente nula (ao nível de 10\%) apenas nos anos 2004 e 2000. Contudo, os coeficientes encontrados nesses anos foram menores que 0,50. Isso invalida o argumento de Jegadeesh (1992) em relação à amostra analisada nesta seção.

TABELA 11 - Alfas dos portfolios L

\begin{tabular}{l|c|c|c|c|c}
\hline & \multicolumn{5}{|c}{ Quintis baseados no Lucro } \\
\hline Ano & 1 & 2 & 3 & 4 & 5 \\
\hline 1998 & -0.0006 & -0.0004 & -0.0051 & $-0.0020^{\star *}$ & -0.0027 \\
\hline 1999 & -0.0003 & 0.0009 & 0.0008 & 0.0002 & 0.0014 \\
\hline 2000 & 0.0001 & $-0.0011^{*}$ & 0.0001 & 0.0002 & 0.0001 \\
\hline 2001 & $0.0010^{\star *}$ & 0.0004 & 0.0013 & 0.0006 & -0.0010 \\
\hline 2002 & $0.0006^{\star}$ & 0.0005 & 0.0005 & $-0.0014 * *$ & $-0.0019 * *$ \\
\hline 2003 & 0.0002 & $0.0006^{\star}$ & -0.0002 & 0.0004 & 0.0002 \\
\hline 2004 & 0.0002 & 0.0004 & 0.0004 & -0.0001 & -0.0005 \\
\hline $1998-2004$ & $0.0004^{\star *}$ & $0.0004^{\star}$ & $0.0017^{\star \star *}$ & -0.0001 & -0.0005 \\
\hline
\end{tabular}

Fonte: Resultados desta pesquisa.

Nota: *significância de 10\% **significância de 05\% *** significância de 01\%

\footnotetext{
${ }^{9}$ A correlação entre a proxy L e o beta não foi calculada a partir do logaritmo da proxy, como nos outros casos, pois o lucro médio do quintil 05 foi negativo.
} 
TABELA 12 - Correlação, ano a ano, entre L e o beta

\begin{tabular}{l|c|c|c|c|c|c|c}
\hline & \multicolumn{7}{c}{ Ano } \\
\hline Proxy & 1998 & 1999 & 2000 & 2001 & 2002 & 2003 & 2004 \\
\hline L & 0.0630 & -0.0420 & $-0.2958^{*}$ & 0.0236 & 0.0255 & -0.1014 & $-0.4011^{\star \star *}$ \\
\hline g. I. & 23 & 36 & 41 & 56 & 60 & 65 & 63 \\
Fonte: Resultados desta pesquisa. \\
Nota: ${ }^{* \star *}$ significância de 1\%
\end{tabular}

\section{CONCLUSÕES}

Os testes de estacionariedade revelaram que todos os ativos da amostra seguem o modelo de passeio aleatório, uma vez que todas as séries temporais de retornos se mostraram estacionárias e aproximadamente $90 \%$ das séries temporais dos preços correntes apresentaram raiz unitária. Sete ações revelaram preços estacionários, mostrando oportunidades de ganho anormal. Contudo, essas ações tiveram baixa liquidez, o que sugere que essas oportunidades não seriam realizáveis. Assim, há evidências de que o mercado brasileiro seja eficiente na forma fraca.

Os resíduos do CAPM tradicional mostraramse heterocedásticos e, no intuito de se contornar esse problema, o CAPM foi estimado novamente a partir da estrutura do modelo GARCH-M. Ao se estimar o CAPM condicional, verificou-se que a maioria dos parâmetros ARCH (1) e GARCH (1) foi significativa ao nível de $10 \%$ de significância. Isso indica que a volatilidade dos portfolios estudados pode ser modelada a partir do modelo GARCH $(1,1)$.

Os testes de efeito tamanho, por sua vez, sugerem que o mercado brasileiro é eficiente na forma semiforte. Não se observou o chamado efeito tamanho ao serem utilizadas as proxies valor de mercado e valor patrimonial, tanto na análise de curto prazo (anual) quanto na de longo prazo (todo o período). Desse modo, pode-se afirmar que a hipótese de Berk (1997), que prediz que apenas o valor de mercado mensuraria um risco relevante, não foi verificada na amostra analisada. Assim, nenhuma proxy de tamanho parece ser fator relevante de risco. Ou seja, independentemente da proxy utilizada, não se identificou o efeito tamanho e isso permite inferir que o mercado acionário brasileiro é informacionalmente eficiente na forma semiforte, no que tange a informações sobre o tamanho das empresas.

Isso, também, significa que os resultados de Romaro (2000), que encontrou evidências de um efeito tamanho invertido utilizando o valor de mercado como proxy, não foram replicados. A amostra por ele analisada revelou que as ações das empresas de maior valor de mercado obtiveram melhor desempenho do que as ações das empresas de baixo valor de mercado. Os resultados aqui apresentados não revelaram efeito tamanho nem para a variável valor de mercado nem para a variável valor patrimonial. Apenas quando se mensurou o tamanho através do lucro, é que se constatou essa anomalia. O "efeito lucro" encontrado teve sentido inverso no longo prazo. Na análise de curto prazo, verificou-se que os quintis das firmas grandes obtiveram retornos anormais apenas em um ou, no máximo, dois anos isolados. $\mathrm{Na}$ análise de longo prazo, observou-se que os retornos anormais desses mesmos quintis foram significativos ao nível de $10 \%$ de significância.

A hipótese de Jegadeesh (1992) a respeito da correlação entre o tamanho das firmas e o beta tampouco foi corroborada pelos dados aqui apresentados. Apenas 9 dos 21 coeficientes de correlação calculados foram significativos ao nível de $10 \%$. Desses 6 revelaram sinal positivo e 3 negativos; e apenas 2 foram superiores a 0,50 em magnitude. Assim, pode-se concluir que realmente não ocorreu o efeito tamanho no período analisado; os testes mostraram que o valor de $\rho_{\beta \mathrm{S}}$ não foi significativo a ponto de ocultar os efeitos da variável tamanho.

Em resumo, os resultados sugerem que o mercado acionário brasileiro é informacionalmente eficiente nas formas fraca e semiforte (no que tange a informações sobre o tamanho das empresas). Enfatize-se que as conclusões se basearam em dados referentes ao período entre 17 de março de 1998 e 3 de agosto de 2004 e não devem ser generalizados.

Por fim, ressalte-se uma limitação dos testes realizados: a freqüência dos dados é apenas diária; o teste não capta, portanto, qualquer turbulência intradia. Considerando que o investidor não espera fechar o dia para atuar no mercado, recomenda-se a implementação de estudos futuros, desenvolvidos com dados intradia, de modo a ampliar os resultados encontrados na presente pesquisa. 


\section{REFERÊNCIAS BIBLIOGRÁFICAS}

ARAÚJO, D. L.; BRESSAN, A. A.; BERTUCCI, L. A.. Análise do Risco de Mercado do Agronegócio Brasileiro: Um Estudo Comparativo entre os Modelos CAPM e GARCH- M. In: Anais do XXVII EnANPAD, Curitiba, 2004.

BANZ, R.. The relantionship between return and market value of common stocks. Journal of Financial Economics, n.9, p. 3-18, 1981.

BERK, J. B.. Does size really matter? Financial Analyst Journal, p.12-18, Set./Out. 1997.

CHAN, K. C.; CHEN, Nai-fu; HSIEH, David A.. An explanatory investigation of the firm size effect. Journal of Financial Economics, 14 , p. $451-471,1985$.

COSTA JUNIOR, N. C. A.; O'HANLON, J.. O efeito tamanho versus o efeito mês-do-ano no mercado de capitais brasileiro: uma análise empírica. Revista Brasileira de Mercado de Capitais, v. 16, p. 61-74, 1991.

COSTA JUNIOR, et al.. Estimação do beta de ações através do método dos coeficientes. In: (Org.). Mercado de capitais: análise empírica no Brasil, São Paulo: Atlas, 2000. (Coleção Coppead de Administração).

DAMODARAN, A.. Avaliação de investimentos: ferramentas e técnicas para a determinação do valor de qualquer ativo. Rio de Janeiro: Qualitymark, 1997.
FAMA, E. F.. Efficient Capital Markets II. Journal of Finance, v. 66, p. 1575-1617, 1991,

FOUSE, W. L.. The small stock's hoax. Financial Analysis Journal, v. 45, n. 4, p. 12-15, Jul/Ago. 1989.

GUJARATI, Damodar N.. Econometria básica. 3. ed. São Paulo: Makron Books, 2000

HAZZAN, S.. Desempenho de ações da Bolsa de São Valores de São Paulo e sua Relação com o Índice Preço-Lucro. 1991. Tese (Doutorado em Finanças) - Escola de Administração de Empresas de São Paulo, Fundação Getúlio Vargas, 1991.

JEGADEESH, N.. Does market risk really explain the size effect? Journal of Financial and Quantitative Analisys, v. 27, n. 3, p. 337351, Set. 1992.

LAMOUNIER, W. M.. Comportamento dos preços no mercado spot de café do Brasil: análise nos domínios do tempo e da freqüência. Prêmio BM\&F de Derivativos 2002. São Paulo, 2003.

LEVINE, D. M.; BERENSON, M. L.; STEPHAN, D.. Estatística: teoria e aplicações usando microsoft excel em português. Rio de Janeiro: LTC, 2000.

ROMARO, P. O efeito tamanho na Bovespa: um estudo sobre os retornos e a volatilidade dos retornos dos portfolios de ações. 2000. Tese (Mestrado em Finanças) - Fundação Getúlio Vargas, Rio de Janeiro, 2000

Univ. Fed. de Minas Gerais

Faculdade de Ciências Econômicas

Rua Curitiba, 832

Belo Horizonte - MG

30170-120 